\title{
Exploration and Application of The Flipped Classroom in College Chinese Teaching
}

\author{
Juan Yang ${ }^{1}$, Tong-ru Wang ${ }^{2}$ \\ 1) Chinese people's armed police force academy, Langfang, Hebei, China \\ ${ }^{2)}$ Chinese people's armed police force academy, Langfang, Hebei, China
}

Abstract-With the continuous renewal of information technology and teaching concept, The flipped classroom arises at the historic moment. It, as a new teaching mode, becomes a hot topic of education at home and abroad and is widely used throughout the world. This article will deeply analyze the flipped classroom's applicaton in college chinese teaching, and put forward feasible and applicable advices combined with specific cases.

Keywords - the flipped classroom, college chinese, application

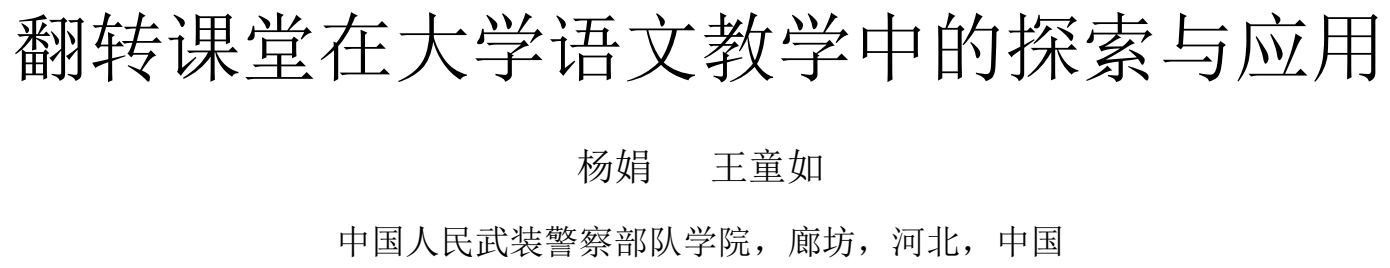

摘 要 随着信息技术和教学理念的不断更新, 翻转课堂应运而生, 其作为一种全新的教学模式, 成为国内外教育界的热门话题, 并在世界范围内被广泛应用。本文将对翻转课堂在大学语文教学中的应用进行深入地分析, 并结合具体案例提出可操作性和应用性的 建议。

关键词 翻转课堂, 大学语文, 应用

\section{1. 引言}

大学语文是提高大学生文化素质教育、人文素质教育 的核心课程之一, 目的在于培养大学生汉语语言文学方面 的阅读、理解、鉴赏、表达能力, 对于提高母语修养及综 合文化素质有着非常重要的作用。大学语文的授课对象是 大学生, 授课内容与方式也更加多元化, 既不是对中学语 文的“补课”, 也不是“高四语文”, 而是较高层次的语文课。 大学语文的培养目标、学科性质, 决定了其教学方法与教 育理念不同于中小学。在新的教学环境下, 翻转课堂的出 现, 为大学语文教学活动拓展了一种新的思路、新的方法。

\section{2. 什么是翻转课堂}

2011 年, 萨尔曼.可汗 (SalmanKhan) 在 TED 大会 上将一种全新的教学方式一“翻转课堂” (the Flipped Classroom) 介绍给全世界, 引起了广大教育工作者的关注。 “翻转课堂 (the Flipped Classroom), 是相对于传统的课堂
上以教师讲授、学生听讲, 课后学生完成作业为主的教学 模式而言的; 它是指课前学生在家观看教学微视频, 完成 知识的理解与把握, 课堂上完成作业, 深化讨论, 动手操 作, 探究创新的课堂教学模式。”翻转课堂打破了传统的教 学模式, 实现了学生的个性化学习, 凸显了学生的学习主 体地位, 也促进了教师教学水平和教育技术能力的提高, 为信息时代的教学改革注入了活力。

\section{3. 翻转课堂的特点与优势}

\section{1 传统教育理念的翻转}

翻转课堂作为一种新兴的教学模式, 颠覆了传统的教 学理念。在传统在传统教学模式下, 课上的学生往往是被 动接受的对象, 整个过程中一直处于“听课”、“记笔记”的 状态, 从而导致在学习过程中缺乏创新能力以及发现问题、 探究问题、解决问题的钻研精神。随着信息时代的到来, 翻转课堂应运而生, 其翻转的是时间、空间、地位、内容, 
实际上是教与学的思维方式的巨大转变。而这种巨大的转 变更具人性化, 为学生提供了个性化的学习空间和学习方 式。

\section{2 教学组织流程的互换}

翻转课堂作为一种新兴的教学模式, 颠倒了传统的教 学过程, 即“教”与“学”的互换。传统课堂是先“教”后“学”, 而翻转课堂是先 “学”后“教”。传统课堂注重地是学习的结 果, 而翻转课堂注重地是学习的过程。学习的过程非常关 键, 例如课堂外的知识传授如何更有效、更能激发学生的 学习兴趣, 课堂内活动如何组织才能促进知识内化等等, 必须重视课堂教学的质量和效果, 而不仅仅是课堂形式的 翻转。这样才能使传统课堂上学生的“齐步走”转变为翻转 课堂的课前“自主学”, 让学生自由掌控自己的学习时间、 内容、方式, 对提高学生的独立思考能力、培养学生的创 新思维大有益处。

\section{3 师生角色的转变}

翻转课堂作为一种新兴的教学模式, 转变了师生角色。 在传统课堂中, 教师是知识的拥有者和传播者, 学生在很 大程度上是被动地接受者。在翻转课堂中, 教师让位于学 生, 把学习的主动权交还给学生, 不再是课堂的中心, 转 变为课堂的组织者、设计者、协调者、指导者。学生摆脱 了被动接受知识的角色, 成为整个教与学过程中的主体。 教师只有努力自我突破, 积极迎接翻转课堂所带来的挑战, 才有可能实现从“主演”到“导演”的转型, 学生只有积极参 与, 变被动为主动, 才能完成“配角”到“主角”的转变。

\section{4. 翻转课堂在大学语文教学中的应用}

语文是门综合性很强的学科, 集工具性、人文性为一 体, 相对于中小学语文侧重知识性与工具性, 大学语文更 注重人文性、审美性, 更注重学生人文素养和审美能力的 培养。大学语文的课堂更需要教师与学生面对面思想的碰 撞、观点的交流、情感的沟通, 让学生带着思考走进课堂, 带着更多人文精神层面的体验感受离开课堂, 而不是单纯 地“听听课”、“记记笔记”。

以华中师范大学出版的《大学语文》第九章中《萧葭》 为教学案例, 运用翻转课堂教学模式进行大学语文课堂教 学改革实践。

\section{1 提前介入一独立学习阶段}

如何设计能调动学生的兴趣, 引发学生思考的教学微 视频成为翻转课堂举足轻重的一环。要针对大学语文的学
科特点, 根据《萧葭》的教学目标、教学重点、难点, 精 心取舍, 整理篎选与《萧葭》有关的资料, 如《萧葭》出 处《诗经》的介绍, 《萧臀》中个别生僻字词的提示, 名家 对《萧葭》主旨的解读, 邓丽君《在水一方》歌曲的展示 等等。创建教学微视频, 营造意境, 让学生融入到诗歌的 情景中, 耳濡目染、润物细无声地接受人文的熏陶。同时 提出思考问题: 《萧臀》一诗的创作主旨历来众说纷纭, 有 “刺襄公”、劝其“遵循周礼”说, “招贤”说, “爱情”说等, 你 更倾向于哪一种解读? 提前介入的不单有学生, 还有教师。 教师可及时掌握学生网上交流平台的互动交流、练习完成 情况, 了解学生的学习理解程度, 并制定个性化指导方案, 为下一阶段的深入学习做好准备, 为知识内化一合作互动 阶段奠定基础。

\section{2 知识内化一合作互动阶段}

(1) 个性化指导

由于教师在课前已经了解掌握学生探讨、交流情况以 及学习效果, 并及时发现了普遍存在的疑难问题和典 型问题, 所以课上针对普遍存在的问题集中讲解, 针 对个别典型问题一对一、面对面独立讲解, 效率高、 目的性强。

(2) 任务布置

在个性化指导的基础上, 汇总梳理出一些有探讨价值 的问题, 供学生合作互动。以《萧臀》为例, 可围绕 如何体会《萧臀》这首诗中的“音乐美”和“朦胧美”展 开研讨, 并拓展阅读李商隐的《锦瑟》、顾城《一代人》。

(3) 合作探究

一千个读者就有一千个哈姆雷特, 可以进行师生、生 生讨论, 畅谈各自对《萧葭》这首诗的形象、意境的 理解, 从“音乐美”当中感受汉语的魅力, 从“朦胧美” 中感受古诗词含蓄慰藉、迷离朦胧的意境, 体会弦外 之音、意外之旨, 获得独特的审美享受与情感体验, 从而树立好学习这首诗的信心。在这个阶段, 需要教 师在学习资源组织、教学策略设计等方面充分考量, 把问题设置、思考启迪有机地结合起来, 从而激发起 学生强烈的主观能动性, 不使翻转课堂流于外在形式。

（4）成果评价

如何检测学生对《萧臀》的学习效果, 提升对《萧臀》 的理解与收获, 课堂即兴演讲或许是一个不错的选择, 即对《萧臀》这首的解读及对自己的启示展示分享。 既梳理知识、提高语言表达能力, 又能实现思想的碰 撞升华, 非常切合大学语文的培养目标。翻转后的课 
堂语文味会更加浓厚, 更加贴近学生的实际生活, 互 动性、主体性更强。

\section{3 资源反馈一巩固提高阶段}

课后教师将课前的视频资料、课上的互动问题、实时 生成的资源以及拓展资料制作成电子压缩包, 在互联网上 与学生共享。电子资料既方便学生随时搜索查阅, 也方便 长期保存。

\section{5. 翻转课堂对于大学语文教学的意义}

随着当代人文科学与自然科学日益交叉渗透, 大学教 育更需要培养出具有全面素质的高质量人才, 翻转课堂的 应用对于大学语文教学意义深远。

\section{1 变“要我学”为“我要学”}

翻转课堂相对于传统课堂, 以课前微视频、课上合作 探究取代了传统的“满堂灌”、“填鸭式”教学模式。观看课 前微视频使学生自主、高效地完成知识的掌握, 这一阶段 有助于学生自控能力、自学能力和探究能力的培养。课上 的合作探究可以使学生更好地与他人交流分享, 对学生的 交流能力、沟通能力和协作能力的提高都大有裨益, 有助 于学生建立科学研究的态度, 学会科学研究的方法。对于 大学生而言, 这种能力的培养比单纯地知识学习更为关键。

\section{2 学生人文素养的丰富}

与其他学科相比, 翻转课堂在大学语文教学中的应用 具有独特的优势。爱尔兰教育家叶芝说过这样一句名言: “教育不是注满一桶水, 而是要点燃一把火”, 对于大学语 文教学来说更是如此。不要小看“这把火”, “星星之火可以 燎原”。“这把火”是培养大学生人文素养的敲门砖, “这把 火”是思想碰撞的火花, 集中体现在翻转课堂的互动性上。

首先, 在教师与学生、学生与学生的交流、沟通、互 动中, 人文、精神层面的提升, 往往才能更深入、更具成 效。翻转课堂教学模式加强了师生、生生互动, 在形式上 打破传统, 淡化了课堂的氛围, 消融了师生身份的隔膜, 拉近了彼此之间心灵的距离。在课上的交流、沟通、互动 中, 不仅让学生受到理性智慧的启迪, 而且可以使学生获 得思想感情、审美体验、精神境界、人生价值多方面的感 悟和感染, 从而使其能更好地传递美感, 陶治情操, 培养 人文素质和审美能力。而人文素质和审美能力的提高有助 于学生形成正确的人生观、价值观, 这一点显得尤为重要。 其次, 语言表达、人际交往能力的培养不可小觑, 其也是 大学语文培养目标中不可缺少的一部分。而这种能力在学
生的互动性、参与性更强, 学生的主体地位更凸显的翻转 课堂上更易培养。

\section{3 教师综合素质的提升}

翻转课堂为师生共同成长提供了更合适的场所, 教师 要从各个方面提升能力素质来承受翻转课堂带来的考验。 在独立学习阶段, 教师需要认真设计制作微视频。信息技 术能力的高低决定了微视频的精彩与否, 人文素养的深浅 决定了微视频的丰富与否, 因此教师要加强学习, 提高自 身信息技术素养, 扩充人文知识储备, 以便学生有效开展 课前的自主学习。在合作互动阶段, 相对传统课堂较单纯 地知识传授对教师有了更高的要求。高质量的问题和活动 设计是翻转课堂有效进行的前提, 这就需要教师不断扎实 学术功底, 丰富知识储备, 提高思维能力, 来应对思维爆 炸的课堂。在巩固提高阶段, 教师做好知识的梳理、总结 和管理, 为后期学生的随机学习提供方便, 为新知识的传 授打牢基础。

\section{6. 结语}

翻转课堂教学模式对于大学语文教学来讲, 既是机遇 又是挑战。翻转课堂的兴起与运用并不是完全替代传统课 堂教学, 无论什么教学模式, 都不可能完美无缺。广大教 育工作者应抓好机遇, 迎好挑战, 应以翻转课堂为契机, 努力探索、不断发展与大学语文教学实际相融合的教学方 法。

\section{参考文献(References)}

[1] Yu TianZhen and Tian Aili, Pursuit and Practice of MOOC \& FLIPPED CASSROOM Teaching Patten---EAST CHINA NORMAL UNIVERSITY MOOC'S Exploration in the Era of Big Data," Primary and Secondary Schools' Information Technology Education, vol. 35, no. 11, pp. 10-13, 2014.

[2] Zhong Xiaoliu, Song Shuqiang and Jiao Lizhen, "Teaching Design Research Based on the Concept of Flipped Classroom Under Informationization Environment," Open Education Research, no. 1,2013.

[3] Zhu Lin, "Flipped Classroom's Exploration and Practice in Undergraduate Teaching," Education and Teaching Forum.vol. 53, no. 12, 2014.

[4] Wang Xiao Dong and Zhang Chenjingzai, "Application and Research of Flipped Classroom in College Teaching," Modern Education Technology, vol. 23, no. 8, 2013. 